\title{
Association of epidermal growth factor receptor and mitogen-activated protein kinase with cystic neoplasms of the pancreas
}

Yuko Kuboki ${ }^{1,2}$, Keiko Shiratori ${ }^{2}$, Takashi Hatori ${ }^{2}$, Izumi Fujita ${ }^{2}$, Akira Kimijima ${ }^{2}$, Masakazu Yamamoto ${ }^{2}$, Makio Kobayashi ${ }^{3,4}$ and Toru Furukawa ${ }^{1,4}$

${ }^{1}$ International Research and Educational Institute for Integrated Medical Sciences, Tokyo Women's Medical University, Tokyo, Japan; ${ }^{2}$ Institute of Gastroenterology, Tokyo, Japan; ${ }^{3}$ Department of Pathology, Tokyo Women's Medical University, Tokyo, Japan and ${ }^{4}$ Department of Surgical Pathology, Tokyo Women's Medical University Hospital, Tokyo, Japan

The molecular pathobiology of pancreatic cystic neoplasms is poorly understood. The aim of this study was to know the involvement of epidermal growth factor receptor (EGFR) and its downstream targets in the serous cystic neoplasms and the mucinous cystic neoplasms of the pancreas. In a total of 72 pancreatic cystic neoplasms, including 39 serous cystic neoplasms and 33 mucinous cystic neoplasms, we examined the expression of native and phosphorylated EGFR, mitogen-activated protein kinase (MAPK), and AKT by immunohistochemistry and somatic mutations in EGFR, KRAS, BRAF, and PIK3CA, by direct sequencing. We also assessed the copy numbers of EGFR transcripts and the amplification of the EGFR gene in some of the samples. We found that EGFR, phosphorylated EGFR, MAPK, and phosphorylated MAPK were evidently expressed in $100,54,100$, and $69 \%$ of the serous cystic neoplasms, and in $12 \%$, none, 33 , and $27 \%$ of the mucinous cystic neoplasms, respectively; the expression was significantly higher and more prevalent in the serous cystic neoplasms than in the mucinous cystic neoplasms. The expression of AKT and phosphorylated AKT was low in both the types of neoplasms. On average, EGFR transcripts in the serous cystic neoplasms and the mucinous cystic neoplasms increased 53.5- and 2.5-fold, respectively, as compared with that in normal tissues, with the increase in the former being significantly greater than that in the latter. Amplification of the EGFR gene was not detected in any of the examined serous cystic neoplasms. None of the tumors had mutations in any of the examined portions of the genes, except two mucinous cystic neoplasms with mutations in codon-12 of KRAS. These results indicate that EGFR and MAPK are actively involved in the pathobiology of serous cystic neoplasms and may therefore be potential diagnostic markers and therapeutic targets in patients with the above mentioned types of neoplasms.

Modern Pathology (2010) 23, 1127-1135; doi:10.1038/modpathol.2010.97; published online 21 May 2010

Keywords: EGFR; MAPK; mucinous cystic neoplasm; PI3K; RAS; serous cystic neoplasm

Cystic neoplasms of the pancreas besides intraductal papillary mucinous neoplasms are classified into two types: serous cystic neoplasms and mucinous cystic neoplasms. Serous cystic neoplasms are usually solitary neoplasms that mostly occur in middle-aged and elderly women. ${ }^{1-3}$ Occasionally,

Correspondence: Professor T Furukawa, MD, PhD, Institute for Integrated Medical Sciences, Tokyo Women's Medical University, 8-1 Kawada-cho, Shinjuku-ku, Tokyo 162-8666, Japan.

E-mail: toru.furukawa@twmu.ac.jp

Received 28 November 2009; revised 4 March 2010; accepted 12 March 2010; published online 21 May 2010 they are enlarged and/or multi-centric, and obstruct the pancreatic duct, thereby causing symptoms., ${ }^{2,4}$ Furthermore, although serous cystic neoplasms are almost entirely benign, some malignant cases that involve the adjacent organs and/or metastasize to the liver have been reported. ${ }^{5-7}$ Patients who have symptoms associated with serous cystic neoplasms usually require surgical treatment. ${ }^{1,2}$ Mucinous cystic neoplasms develop almost exclusively in middle-aged and elderly women, and are associated with a significant risk of malignant change from adenoma to carcinoma. ${ }^{8}$ Therefore, it is generally accepted that mucinous cystic neoplasms need to be 
resected completely. ${ }^{9}$ Patients with non-invasive mucinous cystic neoplasms have a fair prognosis; however, those with invasive mucinous cystic neoplasms have a poor prognosis, with a 5-year survival rate of $16-30 \% .^{10,11}$

Although surgical resection is the treatment of choice for these cystic neoplasms, some patients may not be able to undergo surgery because of their medical condition. Medical treatment is preferred in such cases; however, no effective medicine is available for these cystic neoplasms at present. One of the reasons for this is the lack of knowledge regarding the mechanisms underlying the development and progression of these neoplasms.

The epidermal growth factor receptor (EGFR) is a transmembrane glycoprotein with ligand-inducible tyrosine kinase activity and is one of the four members of the ERBB family. ${ }^{12}$ The ligand-binding EGFR dimerizes and undergoes autophophorylation, which results in the transmission of stimulatory signals to the rat sarcoma viral oncogene homolog (RAS) or phosphatidylinositol-3-kinase (PI3K). ${ }^{12}$ RAS is a GTP-binding molecule that activates signaling cascades, including those related to mitogen-activated protein kinases (MAPKs). ${ }^{13,14}$ PI3K is a membrane-bound lipid kinase that activates the v-akt murine thymoma viral oncogene homolog (AKT). ${ }^{15}$ The RAS-MAPK and PI3K-AKT signaling pathways have essential roles in cellular proliferation and differentiation. Aberrant signaling involving the EGFR and its downstream molecules is implicated in the development of cancers of the head and neck, breast, lung, colon, and pancreas. ${ }^{16-21}$ Recently, EGFRs have come to be regarded as clinically important because of the development of a number of anti-EGFR drugs, including extracellular domain-targeted antibody-based drugs such as cetuximab, panitumumab, and zalutumumab, and intracellular kinase domain-targeted smallmolecule-based drugs such as erlotinib and gefitinib. These drugs enable the medical treatments of some cancers. ${ }^{22}$

In this study, we studied the involvement of EGFR and its related signaling molecules, including KRAS, BRAF, PIK3CA, MAPK, and AKT, in the serous and mucinous cystic neoplasms of the pancreas.

\section{Materials and methods}

\section{Materials}

Seventy-two cystic neoplasms of the pancreas (39 serous cystic neoplasms, 33 mucinous cystic neoplasms) that had been surgically resected at Tokyo Women's Medical University Hospital between 1983 and 2008 were retrospectively analyzed. The clinicopathological features, including the patients' sex and age, and the histological types of the tumors, are shown in Table 1. This study was approved by the ethics committee of Tokyo Women's Medical University.

\section{Immunohistochemistry}

Immunohistochemical staining for the native and phosphorylated forms of EGFR, MAPK, and AKT was performed using formalin-fixed and paraffinembedded tissue sections. The antibodies used were anti-EGFR (DAK-H1-WT; Dako, Glostrup, Denmark), anti-phosphorylated EGFR (DAK-H1-1197; Dako), anti-MAPK (137F5; Cell Signaling Technology Inc., Danvers, MA, USA), anti-phosphorylated MAPK (20G11; Cell Signaling Technology Inc.), anti-AKT (C67E7; Cell Signaling Technology Inc.), and antiphosphorylated AKT (736E11; Cell Signaling Technology Inc.). EGFR and phosphorylated EGFR were manually stained by using CSAII, a biotin-free tyramide signal amplification system (Dako), according to the manufacturer's instructions. MAPK, phosphorylated MAPK, AKT, and phosphorylated AKT were manually stained using a Histofine SABPO kit (Nichirei Corp., Tokyo, Japan), according to the manufacturer's instructions. The primary antibodies against EGFR, phosphorylated EGFR, MAPK, phosphorylated MAPK, AKT, and phosphorylated AKT were diluted to $1: 200,1: 100,1: 250,1: 100$, 1:300, and 1:50, respectively. Staining for EGFR and phosphorylated EGFR was evaluated by using the Zymed evaluation guidelines: score 0, no staining;

Table 1 Characteristics of the examined cases of cystic neoplasms of the pancreas

\begin{tabular}{|c|c|c|c|c|}
\hline & $\operatorname{Sex}(M: F)$ & Mean age & Histological type & No. of cases \\
\hline \multirow[t]{4}{*}{ SCNs } & $7: 32$ & 56.4 & Microcystic serous cystadenoma & 25 \\
\hline & & & Macrocystic serous cystadenoma & 12 \\
\hline & & & Solid serous adenoma & 2 \\
\hline & & & Total & 39 \\
\hline \multirow[t]{4}{*}{ MCNs } & $0: 33$ & 46.3 & Mucinous cystic neoplasm with low- or moderate-grade dysplasia & 29 \\
\hline & & & Mucinous cystic neoplasm with high-grade dysplasia & 2 \\
\hline & & & Mucinous cystic neoplasm with an associated invasive carcinoma & 2 \\
\hline & & & Total & 33 \\
\hline
\end{tabular}

Abbreviations: F, female; M, male; MCN, mucinous cystic neoplasm; SCN, serous cystic neoplasm. 
$1+$, faint or barely perceptible membrane staining; $2+$, moderate membrane staining; and $3+$, strong membrane staining. In this study, scores greater than $1+$ (ie, $2+$ and $3+$ ) were considered to indicate evident expression as described elsewhere. ${ }^{23}$ Staining for MAPK, phosphorylated MAPK, AKT, and phosphorylated AKT was evaluated by comparing the intensities of neoplastic cells with that of islet cells because islet cells show consistent staining in the examined tissues: score 0 , no staining; $1+$, lower-intensity staining; $2+$, equal-intensity staining; and $3+$, greater-intensity staining. The staining characteristics of 500 cells were evaluated and the final score was the sum total of the product of the staining intensity and the corresponding tumor percentage. Total scores that exceeded 100 were considered to indicate evident expression as described elsewhere. ${ }^{24}$

\section{RNA Extraction and Quantification}

Frozen tissues from seven serous cystic neoplasms and eight mucinous cystic neoplasms were used for RNA studies. The frozen sections were prepared and tumor and normal tissue were separately dissected manually from the sections. Because these studied cystic neoplasms were well separated from normal parenchymal tissues, the dissected tissues were composed of neoplastic epithelial cells and supporting stromal cells, the former exceeding far more volume than the latter. Normal parenchymal tissues were composed of acini, ducts, and islets. Total RNA was extracted from the dissected tissues by using the mirVana RNA Isolation kit (Applied Biosystems, Foster City, CA, USA) according to the manufacturer's instructions. Next, $2 \mu \mathrm{g}$ of the extracted total RNA was converted to cDNA by using a High Capacity cDNA Reverse Transcription kit (Applied Biosystems). We determined the copy number of the transcripts by quantitative real-time PCR assays performed using TaqMan Gene Expression Assays and the 7500 Real-Time PCR system (Applied Biosystems), according to the manufacturer's instructions. GAPDH was used as an endogenous reference gene for normalization of expression among the samples. Plasmid clones harboring the TaqMan PCR products were generated using a PCR cloning kit (Stratagene, Cedar Creek, TX, USA) and used as standards for quantitation.

\section{DNA Extraction and Southern Blotting}

Genomic DNA was isolated from the separately dissected tumor and normal tissues obtained from six frozen serous cystic neoplasm specimens as described above by digestion with proteinase-K and extraction with phenol, chloroform, and isoamyl alcohol as described elsewhere. ${ }^{25}$ Southern blotting was performed as follows: $5 \mu \mathrm{g}$ of the genomic DNA was digested with EcoRI or PvuII, electrophoresed on a $0.8 \%$ agarose gel, and transferred onto a nylon membrane as described elsewhere. ${ }^{25}$ Hybridization was performed using the ExpressHyb solution (Takara Bio Inc., Otsu, Japan) and radioactive probes were prepared using a random priming kit (Roche Diagnostics, Basel, Switzerland) containing $\left[\alpha^{-32} \mathrm{P}\right] \mathrm{dCTP}$ (Applied Biosystems), according to the manufacturers' instructions. The templates used for generating the radioactive probes were PCR products harboring exon-19 of the EGFR gene and a unique genomic portion of chromosome-15 amplified from normal human genomic DNA by using paired primers (shown in Supplementary Table 1S).

\section{Mutation Analyses}

Tumor and normal tissues were manually dissected from the formalin-fixed and paraffin-embedded tissues obtained from 39 serous cystic neoplasms and 33 mucinous cystic neoplasms, and used for genomic DNA extraction as described elsewhere. ${ }^{26}$ Somatic mutations in exons 18-21 of EGFR, exon-1 of KRAS, exon-15 of BRAF, and exons 10 and 21 of PIK3CA were analyzed as follows: The extracted genomic DNA was PCR-amplified (primers shown in Supplementary Table 1) according to protocols described elsewhere. ${ }^{27}$ The PCR products were treated with ExoSAP-IT (GE Healthcare, Little Chalfont, UK) according to the manufacturer's instructions. Finally, direct sequencing was performed using a BigDye Terminator (Applied Biosystems) and a 3130x Genetic Analyzer (Applied Biosystems), according to the manufacturer's instructions.

\section{Statistical Analyses}

Statistical analyses were performed using Microsoft Office Excel 2007 (Microsoft Corp., Seattle, WA, USA) or Dr SPSS II (SPSS Inc., Chicago, IL, USA). $P$-values $<0.05$ were considered statistically significant.

\section{Results}

EGFR, MAPK, and AKT Expression in the Cystic Neoplasms of the Pancreas

To know the involvement of EGFR and its downstream targets in serous cystic neoplasms and mucinous cystic neoplasms, the expression of native and phosphorylated EGFR, MAPK, and AKT was immunohistochemically evaluated in 39 serous cystic neoplasms and 33 mucinous cystic neoplasms. Evident expression of EGFR, phosphorylated EGFR, MAPK, and phosphorylated MAPK was seen in $100 \%$ (39/39), 54\% (21/39), 100\% (39/39), and $69 \%(27 / 39)$ of the serous cystic neoplasms, respectively (Figures 1 and 2, and Tables 2 and 3). Interestingly, EGFR expression was quite distinct, 

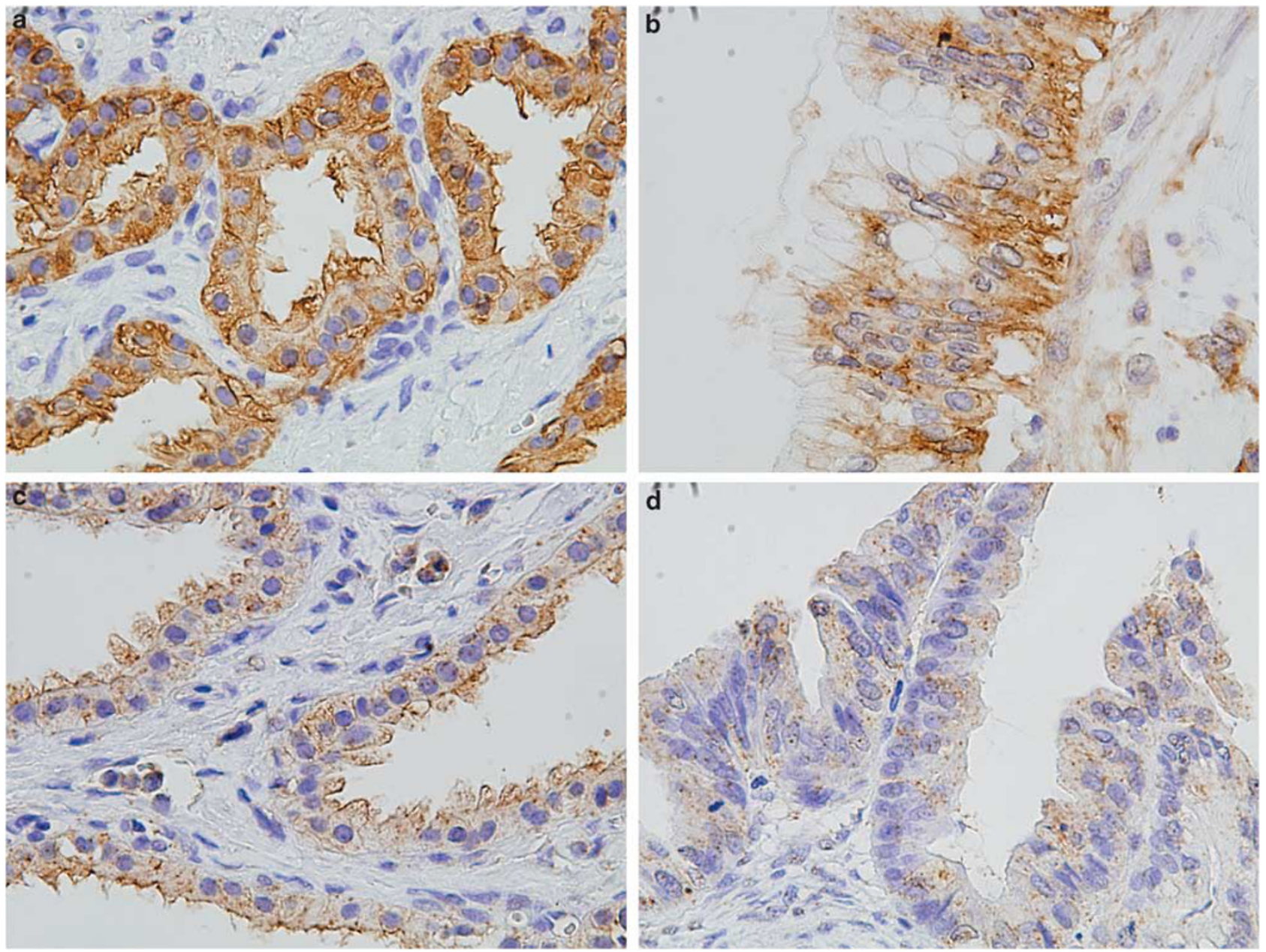

Figure 1 Expression of the native and phosphorylated forms of EGFR in cystic neoplasms, as evaluated by immunohistochemistry. All images have been taken through a $\times 40$ objective lens. Expression of EGFR $(\mathbf{a}, \mathbf{b})$ and phosphorylated EGFR (c, d) in serous cystic neoplasms (a, c) and mucinous cystic neoplasms (b, d), respectively.

and fairly strong membrane staining was observed in most of the serous cystic neoplasms (38/39, 97\%). Expressions of phosphorylated EGFR and phosphorylated MAPK were not associated with each other. In addition, the expressions were not significantly associated with sex, age, and histological type, that is, microcystic, macrocystic, and solid type. Expression of AKT and phosphorylated AKT was not evident in any of the serous cystic neoplasms (Figure 3 and Table 3). Expression of EGFR, phosphorylated EGFR, MAPK, and phosphorylated MAPK was evident in $12 \%$ (4/33), none (0/33), $33 \%(11 / 33)$, and $27 \%(9 / 33)$ of the mucinous cystic neoplasms (Figures 1 and 2, and Tables 2 and 3). The expression was not associated with the histological types of the mucinous cystic neoplasms. The invasive and non-invasive components in the cases of mucinous cystic neoplasms with an associated invasive carcinoma were studied and no difference was found between them. Expression of AKT and phosphorylated AKT was not detected in any of the mucinous cystic neoplasms (Figure 3 and Table 3). Expression of EGFR, phosphorylated
EGFR, MAPK, and phosphorylated MAPK was found to be significantly more frequent and more intense in the serous cystic neoplasms than in the mucinous cystic neoplasms (EGFR, $P<0.0000001$; phosphorylated EGFR, $P<0.0000001$; MAPK, $P<0.0000001$; phosphorylated MAPK, $P=0.0009$; determined using $\chi^{2}$-test with Yates' correction (Tables 2 and 3)). These results indicate that EGFR and MAPK are actively involved in the development and/or maintenance of serous cystic neoplasms.

\section{Mechanisms of EGFR Overexpression}

To elucidate the mechanism of EGFR overexpression in cystic neoplasms, we determined the copy numbers of EGFR transcripts by using quantitative real-time reverse transcription (RT)-PCR analysis of frozen tissues obtained from seven serous cystic neoplasms and eight mucinous cystic neoplasms. The copy number of EGFR transcripts was much higher in all seven serous cystic neoplasms than that in the matched normal tissues (53.5 times higher on 

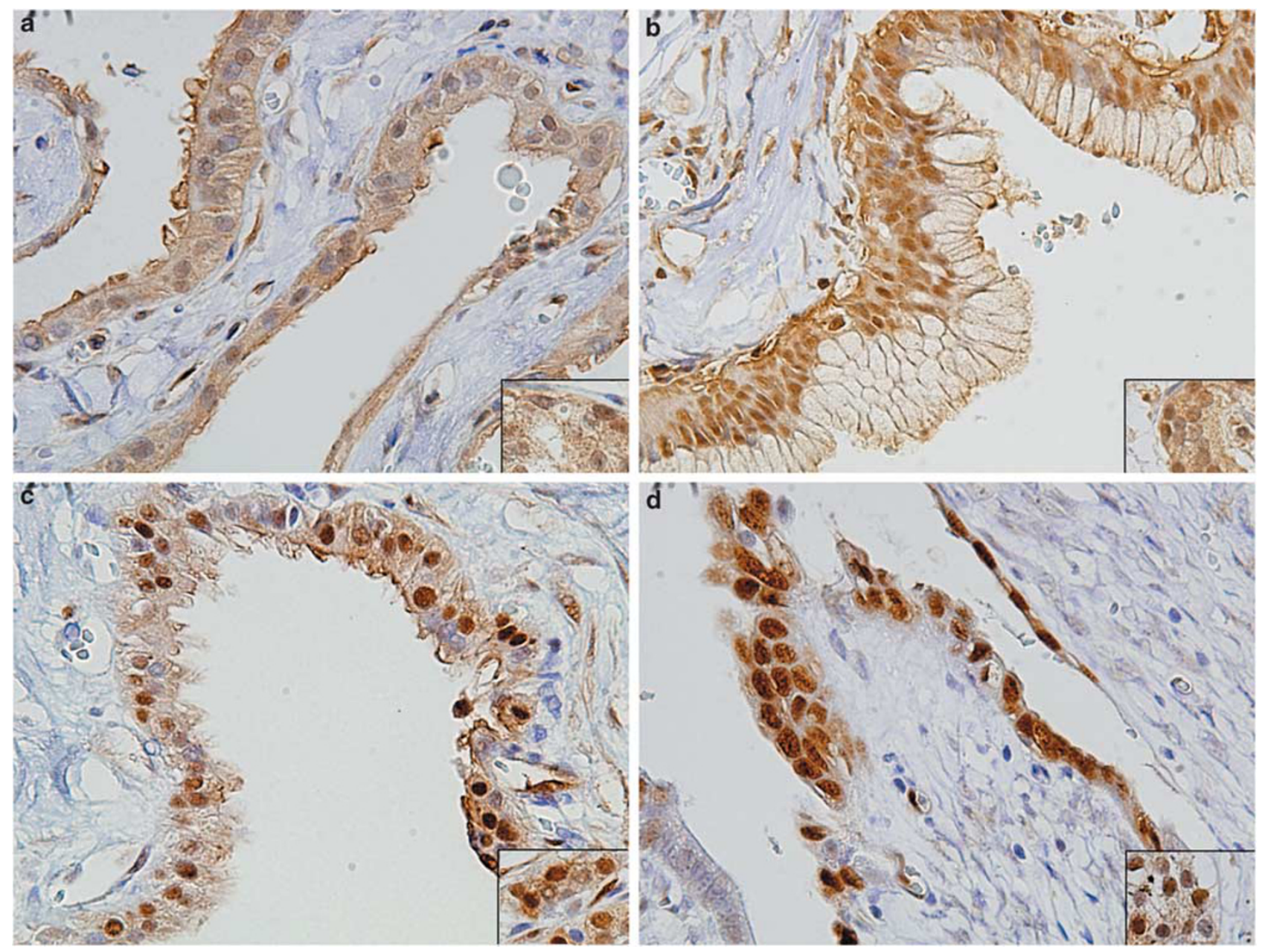

Figure 2 Expression of the native and phosphorylated forms of MAPK in cystic neoplasms, as evaluated by immunohistochemistry. All images have been taken through a $\times 40$ objective lens. Expression of MAPK $(\mathbf{a}, \mathbf{b})$ and phosphorylated MAPK (c, d) in serous cystic neoplasms (a, c) and in mucinous cystic neoplasms (b, d), respectively. The insets show internal control staining in islets, for each stain.

Table 2 Expression of EGFR and phosphorylated EGFR in the cystic neoplasms of the pancreas, as evaluated by immunohistochemistry

Evaluation score

\begin{tabular}{|c|c|c|c|c|}
\hline $3+$, strong $(\%)$ & $2+$, moderate $(\%)$ & $1+$, faint $(\%)$ & 0, none (\%) & $\mathrm{P}$ \\
\hline $38(97)$ & $1(3)$ & $0(0)$ & $0(0)$ & $<0.0000001$ \\
\hline $1(3)$ & $3(9)$ & $2(6)$ & $27(82)$ & \\
\hline $0(0)$ & $21(54)$ & $18(46)$ & $0(0)$ & $<0.0000001$ \\
\hline $0(0)$ & $0(0)$ & $1(3)$ & 32 (97) & \\
\hline
\end{tabular}

Abbreviations: EGFR, epidermal growth factor receptor; MCN, mucinous cystic neoplasm; SCN, serous cystic neoplasm.

${ }^{a}$ Staining intensity was evaluated according to Zymed's evaluation guideline.

average; Figure 4). By contrast, in the mucinous cystic neoplasms, the copy number of EGFR transcripts was only slightly increased than that in the matched normal tissues (2.5 times higher on average; Figure 4). The difference between the increase in the copy number of EGFR transcripts in the case of the serous cystic neoplasm and that in the case of the mucinous cystic neoplasm was significant $(P<0.0000001$; Student's $t$-test $)$. Next, we used Southern hybridization to determine whether the EGFR gene was amplified in the serous cystic neoplasms. EGFR gene amplification was not detected in any of the six serous cystic neoplasms examined (Figure 5). These results indicate that 
Table 3 Expressions of MAPK, phosphorylated MAPK, AKT, and phosphorylated AKT in the cystic neoplasms of the pancreas, as evaluated by immunohistochemistry

\begin{tabular}{lccc}
\hline & \multicolumn{2}{c}{ Staining score $^{\mathrm{a}}$} & \\
\cline { 2 - 3 } & $>100(\%)$ & $\leq 100(\%)$ & $\mathrm{P}$ \\
\hline MAPK & & & \\
SCNs & $39(100)$ & $0(0)$ & $<0.0000001$ \\
MCNs & $11(33)$ & $22(67)$ & \\
Phosphorylated & MAPK & & \\
SCNs & $27(69)$ & $12(31)$ & \\
MCNs & $9(27)$ & $24(73)$ & \\
AKT & & & \\
SCNs & $0(0)$ & $39(100)$ & \\
MCNs & $0(0)$ & $33(100)$ & \\
Phosphorylated & AKT & & \\
SCNs & $0(0)$ & $39(100)$ & \\
MCNs & $0(0)$ & $33(100)$ & \\
\end{tabular}

Abbreviations: MAPK, mitogen-activated protein kinase; MCN, mucinous cystic neoplasm; SCN, serous cystic neoplasm.

${ }^{\mathrm{a}}$ Staining was evaluated by comparing the intensities of neoplastic cells with that of islet cells: score 0 , no staining; 1+, lower-intensity staining; $2+$, equal-intensity staining; and $3+$, higher-intensity staining. The staining characteristics of 500 cells were evaluated and the final score was the sum total of the product of the staining intensity and the corresponding tumor percentage.

EGFR overexpression in the serous cystic neoplasms is associated with an increase in the number of transcripts without amplification of the EGFR gene.

\section{Mutations in EGFR, KRAS, BRAF, and PIK3CA}

We checked for the presence of somatic mutations in the hot spots of EGFR, KRAS, BRAF, and PIK3CA in 38 serous cystic neoplasms (one serous cystic neoplasm sample was excluded because the DNA obtained was of poor quality) and 33 mucinous cystic neoplasms by using direct sequencing. None of the serous cystic neoplasms or mucinous cystic neoplasms had mutations in the examined regions of the genes, except two mucinous cystic neoplasms harboring somatic mutations in codon-12 of KRAS (G12D mutation (GGT to GAT)). Of these, one was a mucinous cystic neoplasm with high-grade dysplasia (cystadenocarcinoma in situ) and this case showed expressions of MAPK and phosphorylated MAPK but no evident expression of EGFR or AKT. The other case was a mucinous cystic neoplasm with low-grade dysplasia (cystadenoma) and this case showed no evident expression of EGFR, MAPK, or AKT. We also found synonymous singlenucleotide polymorphisms in EGFR as follows: ACA in 99 alleles and ACT in 43 alleles at codon629; CAA in 30 alleles and CAG in 112 alleles at codon-787; and ACA in 1 allele and ACG in 141 alleles at codon-725. All of these polymorphisms were found to be registered in the database of single-nucleotide polymorphisms of the National
Center for Biotechnology Information (http:// www.ncbi.nlm.nih.gov:80/).

\section{Discussion}

In this study, we found that serous cystic neoplasms showed high levels of EGFR expression and evident expression of MAPK, whereas mucinous cystic neoplasms showed only a modest level of expression. Overexpression of EGFR in the serous cystic neoplasms was associated with an increase in the number of transcripts without amplification of the EGFR gene. In the case of serous cystic neoplasms, no somatic mutations were detected in any of the examined hot spots of KRAS, BRAF, and PIK3CA. By contrast, somatic mutations were found in codon-12 of KRAS in a fraction of the mucinous cystic neoplasms. These results indicate that EGFR and its downstream target, MAPK, are actively involved in the development and/or maintenance of serous cystic neoplasms.

EGFR is overexpressed in a wide variety of epithelial neoplasms, including pancreatic cancer. ${ }^{16,17}$ With regard to cystic neoplasms of the pancreas, Yeh et $a l^{28}$ and Zhao et $a l^{29}$ independently found that EGFR is infrequently expressed in the mucinous cystic neoplasms. However, there have been no published reports on EGFR expression in the serous cystic neoplasms. Therefore, to the best of our knowledge, our study is the first to show that serous cystic neoplasms frequently overexpress EGFR. EGFR overexpression in cancer is usually associated with amplification of the EGFR gene. In colorectal and breast cancers, amplification of the EGFR gene is invariably associated with overexpression of EGFR. ${ }^{18,19}$ However, cancers that overexpress EGFR do not always show amplification of the EGFR gene, ${ }^{19}$ indicating that amplification of the EGFR gene causes EGFR overexpression, but EGFR overexpression is not always caused by EGFR gene amplification. Our study indicated that EGFR overexpression in the serous cystic neoplasms is associated with an increase in the copy number of transcripts without amplification of the EGFR gene. The increase in EGFR transcripts may be due to the activation of the transcriptional machinery involving complex control systems. ${ }^{30-32}$ The mechanism of the increase in the transcript copy numbers in the serous cystic neoplasms is an important area for further study. Furthermore, it would have been of major interest to test EGFR expressions and gene amplifications in malignant serous neoplasms; however, such malignant cases were not available in this study.

EGFR undergoes autophosphorylation and transmits an active signal to the RAS-MAPK pathway or the PI3K-AKT pathway. ${ }^{12}$ Our study showed that phosphorylated EGFR and phosphorylated MAPK were expressed in a majority of the serous cystic neoplasms, whereas the level of expression of 

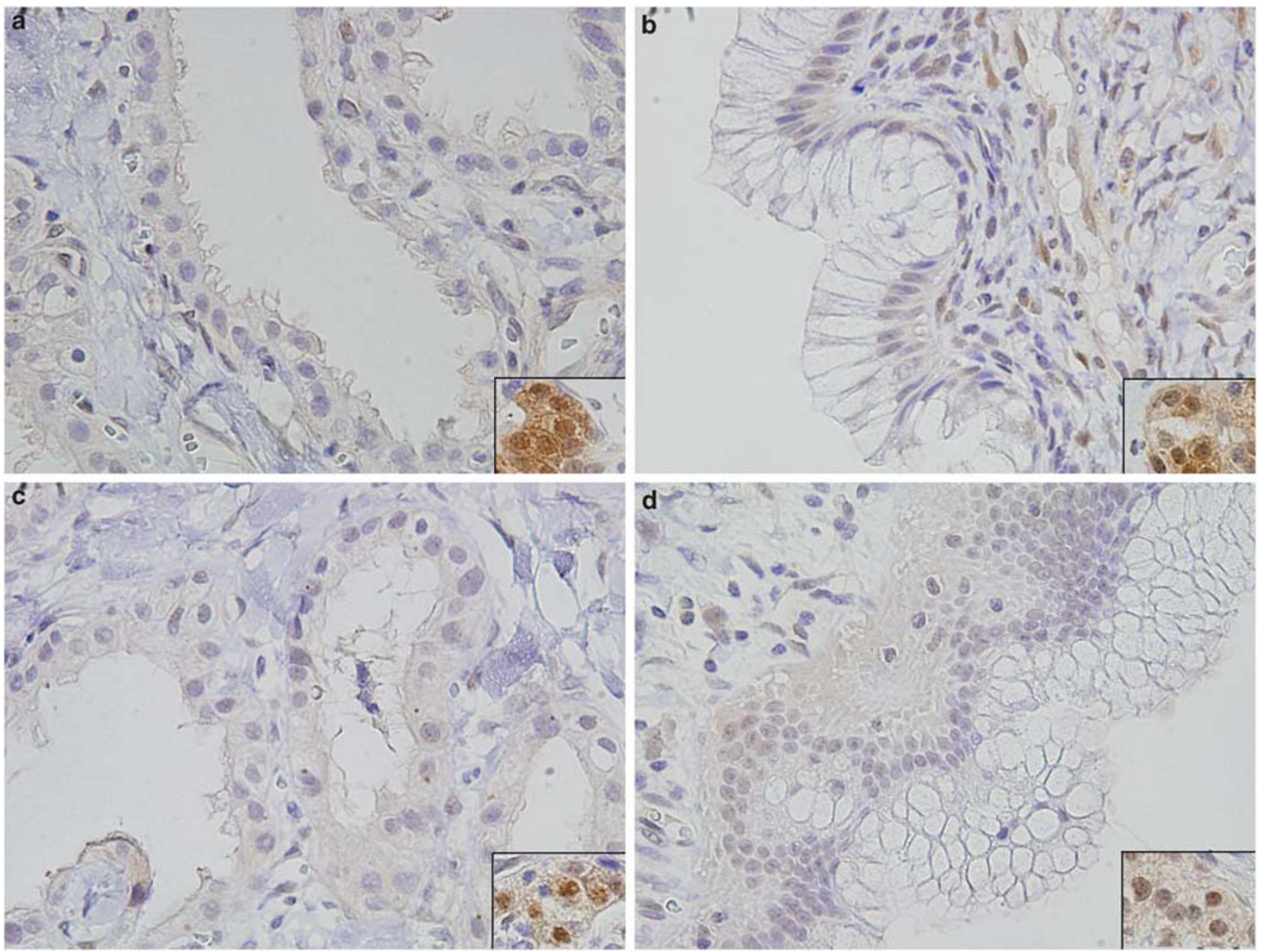

Figure 3 Expression of the native and phosphorylated forms of AKT in cystic neoplasms, as evaluated by immunohistochemistry. All images have been taken through a $\times 40$ objective lens. Expression of AKT $(\mathbf{a}, \mathbf{b})$ and phosphorylated AKT (c, d) expression in serous cystic neoplasms (a, c) and in mucinous cystic neoplasms (b, d), respectively. The insets show internal control staining in islets, for each stain.

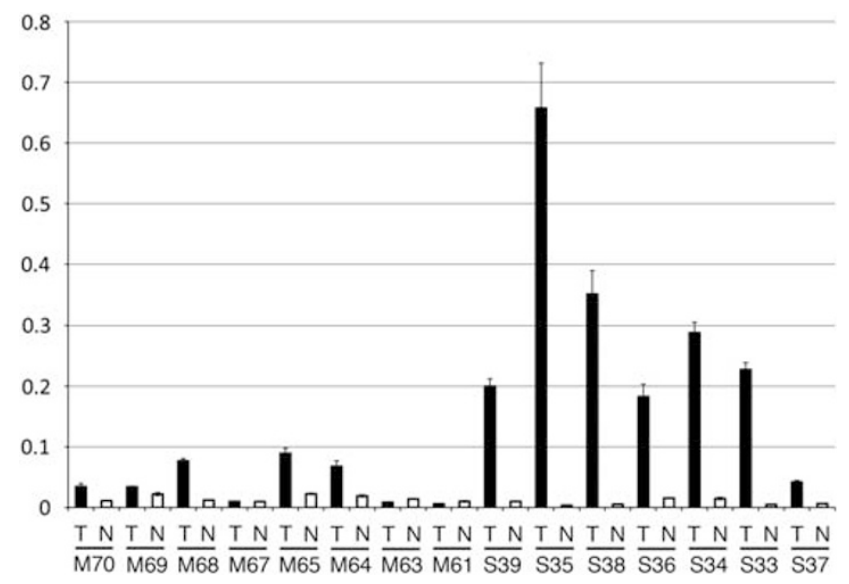

Figure 4 Copy numbers of EGFR transcripts as detected by quantitative real-time PCR. The relative expression of the EGFR transcripts is plotted against the number of $G A P D H$ transcripts in tumor tissues $(\mathrm{T})$ and in matched normal $(\mathrm{N})$ tissues. The sample numbers labeled $\mathrm{M}$ represent the mucinous cystic neoplasms and those labeled S represent the serous cystic neoplasms.

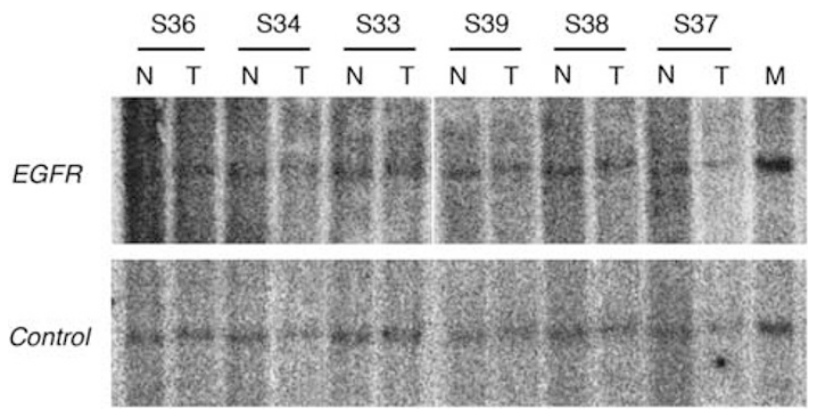

Figure 5 Southern blot analysis of the EGFR gene and a unique genomic portion on chromosome-15 (control) in tumor $(\mathrm{T})$ and matched normal $(\mathrm{N})$ tissues obtained from serous cystic neoplasms. M denotes MIA PaCa2, the pancreatic cancer cell line.

phosphorylated AKT was low; this suggests that activating signals are transmitted from EGFR to the RAS-MAPK pathway rather than to the PI3K-AKT pathway. Furthermore, no gain-of-function mutations were found in any of the hot spots of KRAS, 
$B R A F$, or PIK3CA that were studied, suggesting that EGFR has a primary role in the activation of MAPK in the serous cystic neoplasms. The lack of mutations in KRAS in the serous cystic neoplasms in our study is consistent with the results of a previous study. ${ }^{33}$

Current knowledge indicates that responsiveness to anti-EGFR therapy depends on several molecular features, including amplification of the EGFR gene, overexpression of EGFR, mutation of the EGFR gene, and the absence of self-activating mutations in the signaling molecules downstream from the EGFR gene. In colorectal cancers, EGFR gene amplification is associated with responsiveness to and the survival benefits of anti-EGFR therapy. ${ }^{34}$ In nonsmall-cell lung cancer, EGFR gene amplification, EGFR expression, and somatic mutations in a kinase domain of EGFR are associated with responsiveness to and the survival benefits of anti-EGFR therapy. ${ }^{20,35-37}$ Mutations in the EGFR kinase domain are frequently found in female Asian patients with lung adenocarcinoma who have never smoked,,$^{35}$ but are rarely found in patients with other types of cancers, including pancreatic cancer. ${ }^{38-40}$ Mutations in KRAS or PIK3CA are associated with nonresponsiveness to anti-EGFR therapy. ${ }^{41,42}$ Our study indicated that in the case of serous cystic neoplasms, overexpression of the EGFR protein with increased copy number of EGFR transcripts occurs without amplification of the EGFR gene. The serous cystic neoplasms did not show any mutations in exons 18-23 of EGFR, which encode the kinase domain, nor in any of the examined hot spots in $K R A S, B R A F$, or PIK3CA. These results indicate that further studies are warranted to analyze the potential of EGFR inhibitors as therapeutic agents for serous cystic neoplasms, especially in symptomatic patients in whom it is difficult to perform surgical resection because of complications. In conclusion, EGFR and MAPK are actively involved in the pathobiology of serous cystic neoplasms and may therefore be potential diagnostic markers and therapeutic targets in the case of serous cystic neoplasms.

\section{Acknowledgements}

This work was supported in part by a Grant-in-Aid from the Ministry of Education, Culture, Sports, Science and Technology, Japan; by the Program for Promoting the Establishment of Strategic Research Centers, Special Coordination Funds for Promoting Science and Technology, Ministry of Education, Culture, Sports, Science and Technology, Japan; and by the Pancreas Research Foundation of Japan.

\section{Disclosure/conflict of interest}

The authors declare no conflict of interest.

\section{References}

1 Pyke CM, van Heerden JA, Colby TV, et al. The spectrum of serous cystadenoma of the pancreas. Clinical, pathologic, and surgical aspects. Ann Surg 1992;215:132-139.

2 Tseng JF, Warshaw AL, Sahani DV, et al. Serous cystadenoma of the pancreas: tumor growth rates and recommendations for treatment. Ann Surg 2005;242: 413-419.

3 Klöppel G, Kosmahl M. Cystic lesions and neoplasms of the pancreas. The features are becoming clearer. Pancreatology 2001;1:648-655.

4 Colonna J, Plaza JA, Frankel WL, et al. Serous cystadenoma of the pancreas: clinical and pathological features in 33 patients. Pancreatology 2008;8:135-141.

5 Eriguchi N, Aoyagi S, Nakayama T, et al. Serous cystadenocarcinoma of the pancreas with liver metastases. J Hepatobiliary Pancreat Surg 1998;5:467-470.

6 Friebe V, Keck T, Mattern D, et al. Serous cystadenocarcinoma of the pancreas: management of a rare entity. Pancreas 2005;31:182-187.

7 Gupta R, Dinda AK, Singh MK, et al. Macrocystic serous cystadenocarcinoma of the pancreas: the first report of a new pattern of pancreatic carcinoma. J Clin Pathol 2008;61:396-398.

8 Compagno J, Oertel JE. Mucinous cystic neoplasms of the pancreas with overt and latent malignancy (cystadenocarcinoma and cystadenoma). A clinicopathologic study of 41 cases. Am J Clin Pathol 1978;69:573-580.

9 Garcea G, Ong SL, Rajesh A, et al. Cystic lesions of the pancreas. A diagnostic and management dilemma. Pancreatology 2008;8:236-251.

10 Wilentz RE, Albores-Saavedra J, Zahurak M, et al. Pathologic examination accurately predicts prognosis in mucinous cystic neoplasms of the pancreas. Am J Surg Pathol 1999;23:1320-1327.

11 Sarr MG, Carpenter HA, Prabhakar LP, et al. Clinical and pathologic correlation of 84 mucinous cystic neoplasms of the pancreas: can one reliably differentiate benign from malignant (or premalignant) neoplasms? Ann Surg 2000;231:205-212.

12 Schlessinger J. Common and distinct elements in cellular signaling via EGF and FGF receptors. Science 2004;306:1506-1507.

13 Colicelli J. Human RAS superfamily proteins and related GTPases. Sci STKE 2004;2004:RE13.

14 Boutros T, Chevet E, Metrakos P. Mitogen-activated protein (MAP) kinase/MAP kinase phosphatase regulation: roles in cell growth, death, and cancer. Pharmacol Rev 2008;60:261-310.

15 Stokoe D, Stephens LR, Copeland T, et al. Dual role of phosphatidylinositol-3,4,5-trisphosphate in the activation of protein kinase B. Science 1997;277:567-570.

16 Lemoine NR, Hughes CM, Barton CM, et al. The epidermal growth factor receptor in human pancreatic cancer. J Pathol 1992;166:7-12.

17 Yamanaka Y, Friess H, Kobrin MS, et al. Coexpression of epidermal growth factor receptor and ligands in human pancreatic cancer is associated with enhanced tumor aggressiveness. Anticancer Res 1993;13: $565-569$.

18 Bhargava R, Gerald WL, Li AR, et al. EGFR gene amplification in breast cancer: correlation with epidermal growth factor receptor mRNA and protein expression and HER-2 status and absence of EGFRactivating mutations. Mod Pathol 2005;18:1027-1033. 
19 Shia J, Klimstra DS, Li AR, et al. Epidermal growth factor receptor expression and gene amplification in colorectal carcinoma: an immunohistochemical and chromogenic in situ hybridization study. Mod Pathol 2005;18:1350-1356.

20 Tsao MS, Sakurada A, Cutz JC, et al. Erlotinib in lung cancer-molecular and clinical predictors of outcome. N Engl J Med 2005;353:133-144.

21 Rogers SJ, Box C, Chambers P, et al. Determinants of response to epidermal growth factor receptor tyrosine kinase inhibition in squamous cell carcinoma of the head and neck. J Pathol 2009;218:122-130.

22 Furukawa T. Molecular targeting therapy for pancreatic cancer: current knowledge and perspectives from bench to bedside. J Gastroenterol 2008;43:905-911.

23 Tsiambas E, Karameris A, Lazaris AC, et al. EGFR alterations in pancreatic ductal adenocarcinoma: a chromogenic in situ hybridization analysis based on tissue microarrays. Hepatogastroenterology 2006;53: 452-457.

24 Chadha KS, Khoury T, Yu J, et al. Activated Akt and Erk expression and survival after surgery in pancreatic carcinoma. Ann Surg Oncol 2006;13:933-939.

25 Sambrook J, Fritsch EF, Maniatis T. Molecular Cloning. 2nd edn. Cold Spring Harbor Lab. Press: Woodbury, 1989.

26 Wright DK, Manos MM. Sample preparation from paraffin-embedded tissues. In: Innis MA, Gelfand DH, Sninsky JJ, White TJ (eds). PCR Protocols. Academic Press Inc.: San Diego, 1990, pp 153-158.

27 Yamaguchi H, Shimizu M, Ban S, et al. Intraductal tubulopapillary neoplasms of the pancreas distinct from pancreatic intraepithelial neoplasia and intraductal papillary mucinous neoplasms. Am J Surg Pathol 2009;33:1164-1172.

28 Yeh TS, Jan YY, Chiu CT, et al. Characterisation of oestrogen receptor, progesterone receptor, trefoil factor 1 , and epidermal growth factor and its receptor in pancreatic cystic neoplasms and pancreatic ductal adenocarcinoma. Gut 2002;51:712-716.

29 Zhao J, Liang SX, Savas L, et al. An immunostaining panel for diagnosis of malignancy in mucinous tumors of the pancreas. Arch Pathol Lab Med 2001;125: 765-769.

30 Zheng ZS, Goldsmith LA. Modulation of epidermal growth factor receptors by retinoic acid in ME180 cells. Cancer Res 1990;50:1201-1205.

31 Alsat E, Haziza J, Scippo ML, et al. Increase in epidermal growth factor receptor and its mRNA levels by parathyroid hormone (1-34) and parathyroid hormone-related protein (1-34) during differentiation of human trophoblast cells in culture. J Cell Biochem 1993;53:32-42.

32 Sizemore N, Choo CK, Eckert RL, et al. Transcriptional regulation of the EGF receptor promoter by HPV16 and retinoic acid in human ectocervical epithelial cells. Exp Cell Res 1998;244:349-356.

33 Ishikawa T, Nakao A, Nomoto S, et al. Immunohistochemical and molecular biological studies of serous cystadenoma of the pancreas. Pancreas 1998;16:40-44.

34 Moroni M, Veronese S, Benvenuti S, et al. Gene copy number for epidermal growth factor receptor (EGFR) and clinical response to anti-EGFR treatment in colorectal cancer: a cohort study. Lancet Oncol 2005;6: 279-286.

35 Paez JG, Janne PA, Lee JC, et al. EGFR mutations in lung cancer: correlation with clinical response to gefitinib therapy. Science 2004;304:1497-1500.

36 Cappuzzo F, Hirsch FR, Rossi E, et al. Epidermal growth factor receptor gene and protein and gefitinib sensitivity in non-small-cell lung cancer. J Natl Cancer Inst 2005;97:643-655.

37 Takano T, Ohe Y, Sakamoto H, et al. Epidermal growth factor receptor gene mutations and increased copy numbers predict gefitinib sensitivity in patients with recurrent non-small-cell lung cancer. J Clin Oncol 2005;23:6829-6837.

38 Mimori K, Nagahara H, Barnard GF, et al. Epidermal growth factor receptor gene is highly conserved in pancreatic cancer cells. Pancreas 2005;31:299.

39 Immervoll H, Hoem D, Kugarajh K, et al. Molecular analysis of the EGFR-RAS-RAF pathway in pancreatic ductal adenocarcinomas: lack of mutations in the BRAF and EGFR genes. Virchows Arch 2006;448: 788-796.

40 Tzeng CW, Frolov A, Frolova N, et al. Epidermal growth factor receptor (EGFR) is highly conserved in pancreatic cancer. Surgery 2007;141:464-469.

41 Linardou H, Dahabreh IJ, Kanaloupiti D, et al. Assessment of somatic k-RAS mutations as a mechanism associated with resistance to EGFR-targeted agents: a systematic review and meta-analysis of studies in advanced non-small-cell lung cancer and metastatic colorectal cancer. Lancet Oncol 2008;9: 962-972.

42 Sartore-Bianchi A, Martini M, Molinari F, et al. PIK3CA mutations in colorectal cancer are associated with clinical resistance to EGFR-targeted monoclonal antibodies. Cancer Res 2009;69:1851-1857.

Supplementary Information accompanies the paper on Modern Pathology website (http://www.nature.com/ modpathol) 